\title{
WEGEN UNSERER SÜNDEN GESTORBEN? NEUE ZUGÄNGE ZUR SOTERIOLOGISCHEN BEDEUTUNG DES TODES JESU*
}

\author{
Because of our Sins? NeW PERSPECTIVES ON THE \\ SOTERIOLOGICAL MEANING OF JESUS' DEATH
}

\author{
Morto por Nossos PeCAdos? Novas PERSPECTIVAS DO \\ SIGNIFICADO SOTERIOLÓGICO DA MORTE DE JESUS
}

\section{Leandro Luis Bedin Fontana**}

\section{RESUMO}

O artigo retoma a famosa controvérsia entre os renomados teólogos alemães Magnus Striet e Jan-Heiner Tück acerca do sentido soteriológico da morte de Jesus, ocorrida em 2012, e procura avançar na reflexão teológica. Tendo em vista alguns desenvolvimentos anômalos do discurso cristão sobre a morte de Jesus como a hipostatização da dor e do sofrimento, o artigo sugere uma criteriologia para tratar de temas teológicos pertinentes a essa questão tais como as noções de expiação, pecado e vicariedade. O critério decisivo para uma hermenêutica adequada do sentido soteriológico da morte de Jesus é - assim propõe o artigo - a unidade entre a pluralidade de interpretações neotestamentárias de sua morte e o relato de sua vida e de sua mensagem.

Palavras-chave: Morte de Jesus. Salvação. Expiação. Pecado. Vicariedade.

\footnotetext{
* Gekürzte und veränderte Version meiner am 16.02.2016 an der PhilosophischTheologischen Hochschule Sankt Georgen gehaltenen Probevorlesung als Teilbedingung zur Erlangung des Doktortitels.

** Licenciado em Filosofia (2001) pela FAFIMC, Viamão, RS, bacharel em Teologia (2008) pela Pontifícia Universidade Católica do Rio Grande do Sul e doutor em teologia (fevereiro de 2016) pela Philosophisch-Theologische Hochschule Sankt Georgen, Frankfurt, Alemanha, com uma tese sobre o diálogo inter-religioso cujo título é: Freed by Love: Rethinking the Soteriological Premises of the Christian Discourse on the Interreligious Dialogue in the Light of Jesus' Message and Practice of Love. Atualmente trabalha como pesquisador bolsista do Programa Nacional de Pós-Doutorado, vinculado ao Programa de Pós-Graduação em Teologia da PUCRS.
}

\begin{tabular}{|l|l|l|l|l|l|}
\hline Teocomunicação & Porto Alegre & v. 46 & n. 1 & p. 104-122 & jan.-jun. 2016 \\
\hline
\end{tabular}




\section{ABSTRACT}

This article takes up the famous controversy occurred in 2012 between the renowned German theologians Magnus Striet e Jan-Heiner Tück about the soteriological meaning of Jesus' death and seeks to progress in the theological reflection. In view of some anomalous developments of the Christian discourse on Jesus' death like the hypostatization of pain and suffering, the article suggests guiding principles to deal with theological topics related to this issue such as atonement, sin, and vicariousness. Accordingly, the decisive criterion for a proper hermeneutics of the soteriological meaning of Jesus' death is the unity between the plurality of new-testamental interpretations of his death and the account of his life and message.

Keywords: Jesus' Death. Salvation. Atonement. Sin. Vicariousness.

\section{$1 \quad$ Einführung}

Wie der Dogmatiker Klaus Vechtel in seinem Artikel „Musste Jesus für uns leiden"1 richtig beobachtete, stieß das lebhafte, im Jahr 2012 geführte Streitgespräch ${ }^{2}$ zwischen den renommierten Dogmatikern Magnus Striet und Jan-Heiner Tück die Debatte um die soteriologische Bedeutung des Todes Jesu im deutschsprachigen Raum neu an. Da es sich dabei um nicht weniger als eine der Kernaussagen des soteriologischen Kerygmas des Christentums handelt, die im Laufe der Geschichte eine fast undurchschaubare Vielfalt an theologischen Deutungsmöglichkeiten hervorrief, sind die andauernden hermeneutischen Bemühungen der Dogmatik wieder einmal angefragt.

Denn es ist nicht gleichgültig, ob Sühne darin besteht, dass Gott für die Sünden der Menschen sühnt oder eben darin, dass er ,,[...] in der Menschwerdung die Satisfaktion für seine eigene Schöpfungstat [leistet], indem er sich als Sohn das zumutete, was er allen Menschen zumutet." ${ }^{3}$ Dabei geht es freilich nicht an erster Stelle um eine fundamentaltheologische Plausibilisierung dieses Glaubenssatzes der plural und säkular gewordenen Welt gegenüber, sondern primär um binnentheologische Erklärungsversuche des verbindlichen

\footnotetext{
VECHTEL, K. Musste Jesus für uns leiden?, S. 341-359.

2 Dieses am 8. Februar 2012 in München geführte Streitgespräch wurde von der Katholischen Akademie in Bayern veranstaltet.

3 STRIET, M. Erlösung durch den Opfertod Jesu?, S. 23.
} 
Gehalts solcher Aussagen für einen in der heutigen Welt gelebten und verantwortbaren Glauben.

Im Zentrum dieses Aufsatzes stehen drei Kernfragen der herkömmlichen Deutungsversuche des Todes Jesu, die nicht zuletzt anlässlich der oben erwähnten Debatte in der deutschsprachigen Theologie erneut gestellt wurden: die die Kritik Navid Kermanis aufgreifende, ${ }^{4}$ von Striet infrage gestellte Hypostasierung des Leidens und der Gewalt; das übermäßige Gewicht der Sünde in traditionellen Erlösungstheologien; und der Konflikt zwischen etlichen Stellvertretungstheorien und subjekttheoretischen Ansätzen. Die aufgestellte These ist, dass die entscheidenden Kriterien für jegliche theologische Deutung des heilsamen Todes Jesu sein Leben und seine Botschaft sind. Begründet wird sie durch die Analyse einiger geschichtlicher Fehlentwicklungen des christlichen Erlösungsglaubens, welche vor allem durch eine Verselbständigung des Todes Jesu und eine Hypostasierung seines Leidens zustande kamen, d.h. durch eine Trennung zwischen Leben und Botschaft einerseits und heilsamem Tod andererseits.

\section{Der Umgang mit den Zeugnissen des Neuen Testaments}

Auf eine ausführliche Untersuchung und Darlegung des biblischen Zeugnisses vom Tod Jesu muss an dieser Stelle aufgrund des begrenzten Umfangs dieses Artikels verzichtet werden. Es gibt jedoch auch einen schlichten, sachlichen Grund. Auf die Frage nach dem Sinn und der Bedeutung des Todes Jesu gibt nämlich das Neue Testament ,[...] nicht nur eine Antwort, sondern eine ganze Reihe von Antworten", 5 die von einer ständigen Bemühung der frühen Christen, diesen Tod neu zu verstehen, zeugen. „Sie alle sind Versuche, das Handeln Gottes in Jesu Tod zur Sprache zu bringen, Sprachversuche, die nicht mit der Sache selbst verwechselt werden dürfen." ${ }^{\text {"6 }}$

4 KERMANI, N. Bildansichten. Mit einem Hinweis auf diesen Artikel fängt Striet seinen Aufsatz über den Tod Jesu an.

5 BARTH, G. Der Tod Jesu Christi im Verständnis des Neuen Testaments, S. 157, Hervorhebung im Original; vgl. auch STEYN, G. Soteriological Perspectives in Luke's Gospel, S. 67-99.

6 BARTH, G. Der Tod Jesu Christi im Verständnis des Neuen Testaments, S. 157, Hervorhebung im Original. 
Freilich bedeutet das nicht, dass der Theologe bzw. die Theologin sich aus diesen diejenige Antwort aussuchen sollte, die ihm oder ihr zufällig am besten passt und einleuchtet. Im Neuen Testament stehen sie nebeneinander und sind nicht selten miteinander verbunden. Dadurch ergänzen und korrigieren sie sich gegenseitig. Die Aufgabe des Theologen bzw. der Theologin besteht ebenso heute wie auch in neutestamentlichen Zeiten eben darin, das pro nobis und das extra nos dieses wunderbaren Ereignisses zu begreifen, das offenbarte Wort Gottes in menschliche Sprache zu bringen. Selbstverständlich besitzen die neutestamentlichen Bilder und Metaphern einen herausragenden Stellenwert, doch darf die theologische Reflexion über dieses Heilsgeschehen von keinem von ihnen im Besonderen bestimmt werden. Die Bemühungen des Theologen bzw. der Theologin richten sich nicht exklusiv an diesen Bildern aus, wenn sie auch maßgebend und konstitutiv für den Glauben sind, sondern am Jesus-Ereignis als ganzem, gleichwohl seine Interpretation zum großen Teil von ihnen, also den Bildern und Metaphern des Zeugnisses über und von Jesus, geprägt wird.

\section{Geschichtliche Deutungsversuche}

Ein Beleg dafür, dass das Neue Testament nicht die einzigen autorisierten Bilder und Metaphern für die Deutung des Todes Jesu liefert, ist die Vielfalt an Deutungsversuchen dieses geheimnisvollen Todes, die die christliche Tradition durchdringt. Ein kurzer Überblick über die wichtigsten dieser Ansätze bietet sich hier deshalb an.

Die christlichen Theologien des 2. und 3. Jahrhundert lassen sich durch einen Weltpessimismus kennzeichnen, der bereits in den johanneischen Schriften erkennbar wird. Es ist nicht zuletzt die Zeit der Christenverfolgung, die den Eindruck verstärkt, dass die Welt nichts anderes als „Herrschaftsbereich des Bösen“ sei, ein Ort, wo der Mensch hilflos bösen Mächten, Dämonen, ja dem Teufel, ausgeliefert ist. Theologen wie z.B. Irenäus von Lyon und Justin der Märtyrer versuchen deshalb, ihren Gemeinden die Gewissheit zu vermitteln, dass die konkret erfahrbare Macht der Dämonen letzten Endes nichts mehr als ein hilfloses Rebellieren sein kann, da der Sieg über sie schon errungen ist durch die Erlösungstat Jesu Christi. ${ }^{7}$

WERBICK, J. Soteriologie, S. 146-147; vgl. auch STUDER, B. Gott und unsere Erlösung im Glauben der Alten Kirche, S. 67-69. 
Bei Origenes (gest. 253/4) und Augustinus verbreitet sich die Lehre der Zahlung eines Lösepreises, welche bereits bei Irenäus zu finden war, der zufolge der Sieg Christi in der „Erstattung eines gerechten Lösepreises an den Teufel" bestehe. ${ }^{8}$ Denn der Teufel, so die Theorie, habe der Sünde des Menschengeschlechts wegen ein Anrecht auf alle Menschen. Da Gott selbst dem Teufel Gerechtigkeit widerfahren lassen wolle, könne dieses Anrecht nur gegen die Bezahlung eines Lösepreises abgelöst werden, des Preises seines eigenen Sohnes. Gott habe beschlossen, nach Augustinus, den Teufel ,nicht durch Macht, sondern durch Gerechtigkeit zu besiegen".9 Dieser sogenannten Teufelsbetrugstheorie zufolge musste dann der Teufel der Gerechtigkeit willen alle von ihm festgehaltenen Schuldner entlassen, die an Christus glaubten. Weil Christus durch seine göttliche Macht die Fessel des Todes zerrissen und das Tor des Hades aufgerissen habe, besiege er den Teufel, dessen Macht zurückerobert worden sei.

Die Theorie des Lösegeldes erfuhr bereits vor Augustinus etwa bei Gregor von Nazianz (gest. 390) harsche Kritik. Noch härterer Kritik wird sie jedoch später bei Anselm von Canterbury begegnen, der nach dem Münsteraner Theologen Jürgen Werbick ,[...] seine Satisfaktionslehre als Gegenentwurf gegen die ,Teufelsbetrugshypothese" [verstanden habe]." Anselm will sowohl Gottes Prädikaten der Gerechtigkeit, Allmacht und Güte als auch der Freiheit des Menschen gerecht werden. Da aber die Ehre Gottes durch die Sünde verletzt worden sei, müsse er entweder den Menschen vernichten durch Strafe (aut poena) oder eine die Sünde sühnende Ersatzleistung erbringen (aut satisfactio = Wiedergutmachung). Auch wenn die neuere Anselm-Forschung behaupten mag, es gehe nicht um eine „narzisstische“ Ehre Gottes, sondern um den ,universalen ordo des Rechts und des Friedens zwischen Gott und Mensch" ${ }^{10}$ und darum, dass die Freiheit des Sohnes unbeschränkt bleibe, drängt sich dennoch die Frage auf, ob nicht der Tod Jesu nach wie vor als Bedingung des Sündennachlasses zu denken wäre, was wiederum doch einen Einschnitt in die Freiheit Gottes darstellte.

Eine solche Kritik an Anselms Theorie wird nicht nur heute geübt, sondern bereits von scholastischen Theologen wie Thomas von Aquin.

\footnotetext{
8 WERBICK, Soteriologie, S. 149.

9 De Trinitate XIII, 17 und 18.

${ }^{10}$ VECHTEL, K. Musste Jesus für uns leiden?, S. 342.
} 
Für ihn hätte die Allmacht Gottes durchaus andere Wege wählen können, um den Menschen von Sünde und Schuld zu erlösen. ${ }^{11}$ Statt sich aber dem Nachdenken über solche Alternativwege zu widmen, erweiterte der Scholastiker den Horizont der Debatte auf die Heilsbedeutung der Inkarnation und der Mysterien des Lebens Jesu, die in die Reflexion über den Tod und Auferstehung mündete. Er verkürzte allerdings die Soteriologie nicht auf die Engführung einer Kreuzestheologie. ${ }^{12}$ Die Antwort auf Anselms Frage Cur Deus homo lautet bei Thomas: weil Gott sich den Menschen auf menschliche Weise mitteilen wollte (se aliis communicare). ${ }^{13}$ Darin bestehe der Sinn der Inkarnation ,[...] die den Graben zwischen Gott und den Menschen überbrückt und Gottesfreundschaft ermöglicht." ${ }^{\text {"14 }}$

Im Laufe seiner Auseinandersetzung mit der scholastischen Theologie stellt Martin Luther einer solchen theologia gloriae seine theologia crucis entschieden gegenüber. „Christi Leiden muß nicht mit Worten und Schein, sondern mit dem Leben und wahrhaftig gehandelt werden. " ${ }^{15}$ Luther war eher skeptisch gegenüber einer Wortweisheit, die Gerhard Ebeling heute mit hermeneutischer Theologie gleichsetzt, da

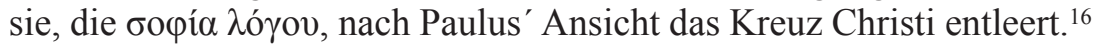
Durch Luther kehren dementsprechend der augustinsche Leitgedanke der ursprünglichen Sündhaftigkeit des Menschen sowie Paulus' Theologie des Kreuzes als Offenbarungsgeschehen wieder in den Mittelpunkt.

Um einen kompakten Überblick über die Vielfalt an soteriologischen Ansätzen des 20. Jahrhunderts zu erleichtern, schlug der Bonner Theologe Karl-Heinz Menke eine dreifache Typisierung für die zentralen Denkformen der Soteriologie dieser Zeit vor, nämlich Stellvertretung, Befreiung, Communio. ${ }^{17}$ Was diese verschiedenen Typen unterscheidet, ist vor allem die Verhältnisbestimmung von göttlichem und menschlichem Erlösungshandeln. Während beim christozentrischen Modell der Stellvertretung das Erlösungswerk ausschließlich vom Erlöser vollbracht wird, verlangt das anthropozentrische Modell (Befreiung)

\footnotetext{
${ }_{11}$ VECHTEL, K. Musste Jesus für uns leiden?, S. 342.

12 S. th. III, q. 46, art. 2 ad 3.

13 TÜCK, J.-H. Am Ort der Verlorenheit, S. 50.

${ }^{14}$ S. th. III, q. 1, art. 1.

15 TÜCK, J.-H. Am Ort der Verlorenheit, S. 50-51.

16 WA 2; 141,37-142,1.

${ }^{17}$ EBELING, G. Der Sühnetod Christi als Glaubensaussage, S. 3.
} 
„[...] den Verzicht auf jede Aussage über eine Erlösung unabhängig von der befreienden Praxis der Erlösten. "18 Nach dem Alternativmodell der Communio hingegen, repräsentiert vor allem durch Gisbert Greshake in Auseinandersetzung mit den Gründern der sogenannten „Integrierten Gemeinde" Ludwig Weimer, Rudolf Pesch und Gerhard Lohfink, vollzieht sich das Erlösungshandeln durch die ,[...] Abbildung der von Christus im Heiligen Geist gelebten Communio mit dem Vater durch die Christen [...]." ${ }^{19} \mathrm{Zu}$ den prominentesten Vertretern des Stellvertretungsmodells zählen Namen wie Karl Barth, Hans Urs von Balthasar und Raymond Schwager, wohingegen das Befreiungsmodell von Theologen wie Johann Baptist Metz und Hans Kessler sowie von Befreiungstheologen entwickelt wurde.

\section{Systematische Überlegungen}

Gleichgültig, wie man den Tod Jesu soteriologisch auslegt, was im Zentrum dieser biblischen Glaubensaussage steht, ist die Ermöglichung unbedingter, ja einer „,neuen“"Freiheit. In der Tat meint der christliche Begriff der Erlösung in seinem Ursprung, d.h. in Übereinstimmung mit den neutestamentlichen Zeugnissen, so tendenziös und ideologisch diese Wörter heute klingen mögen, unbedingte, vollkommene Freiheit, Rettung, Befreiung von jeglichem Zwang. Außerdem bringt eine jede soteriologische Deutung dieses Todes die Aspekte des ,pro nobis“" und des ,extra nos“" zum Ausdruck.

\subsection{Die Verselbstständigung des Sühnemotivs}

Problematisch erscheint hingegen eine „Verselbstständigung des Sühnemotivs", wie es Thomas Pröpper auf den Punkt gebracht hat, ${ }^{20}$ welche nicht zuletzt durch den Einfluss der anselmschen Satisfaktionslehre die westliche Theologie maßgeblich prägte. Bereits Hans Kessler hatte auf die Tatsache aufmerksam gemacht, dass eine soteriologische Fixierung entweder auf die Inkarnation oder auf den Tod Jesu ,das entscheidende ,Zwischenstück“" ausspare und deswegen der Gesamtheit der Offenbarung Gottes nicht gerecht werden könne. ${ }^{21}$ Die

\footnotetext{
${ }^{18}$ MENKE, K.-H. Stellvertretung - Befreiung - Communio, S. 21-59.

${ }^{19}$ MENKE, K.-H. Stellvertretung - Befreiung - Communio, S. 52.

${ }^{20}$ MENKE, K.-H. Stellvertretung - Befreiung - Communio, S. 52.

${ }^{21}$ PRÖPPER, Th. Erlösungsglaube und Freiheitsgeschichte, S. 78.
} 
Ausblendung des Lebens und der Botschaft Jesu zugunsten der „bloßen“ Verkündigung des Kerygmas, so wie es etwa Bultmann vorschlug, bedeutete m.E. hinsichtlich Gottes Selbstmitteilung die Entbehrung jeglicher Kriterien, anhand derer überhaupt Aussagen über den Tod Jesu gemacht oder als bewährt erwiesen werden können. Darüber hinaus suggeriert dies eine Trivialisierung des Ernstes des Lebens Jesu.

Jesus will Israel doch aufrufen, das jetzt ergehende Gnadenangebot Gottes anzunehmen, sich von der unbegreiflich großen, allem menschlichen Tun des Gesetztes zuvorkommenden Vaterliebe Gottes Vergebung der Sünden schenken zu lassen, um das Heil der Gottesherrschaft zu erben. Hätte er diese Aufgabe ,guten Gewissens" - man muß schon sagen, mit jener Unbedingtheit, die die Evangelien gewiß zu Recht bekunden - in Angriff nehmen können, wenn er doch im voraus wußte, daß dies Gnadenangebot in Wirklichkeit gar nicht das letzte und endgültige Wort Gottes zum Thema Sünde und Heilerlangung ist, daß Gott nämlich noch eine andere Möglichkeit der Sündenvergebung vorausbestimmt hat?22

In Folge dieses Gedankenganges Vögtles stimmt ihm deswegen Pröpper zu, „[...] dass gegenüber der von Jesus verkündeten Sündenvergebung die Entsündigung aufgrund stellvertretender Sühne „ein strukturell neuer Modus der Sündenvergebung“ gewesen wäre $[\ldots] .{ }^{، 23}$

\subsection{Die Gefahr eines problematischen Verständnisses des Begriffs der Sünde}

Darum ist für eine systematische Entfaltung des Sündennachlasses letztendlich die Definition von Sünde selbst entscheidend. Wird der Sünde das ,unendliche Gewicht" ${ }^{24}$ beigemessen, das der Verletzung der Ehre eines unendlichen Gottes entspricht, so wie bei Anselm, oder fasst man Sünde als eine ursprüngliche, ontologische Bestimmung des menschlichen Daseins wie bei Paulus oder noch stärker bei der Erbsündenlehre des Augustinus auf, so bedarf es tatsächlich eines von außerhalb der Geschichte kommenden Erlösers, der allein kraft seines Werkes den Menschen aus dieser Macht retten und neues, göttliches

\footnotetext{
${ }^{22}$ VÖGTLE, A. Todesankündigungen und Todesverständnis Jesu, S. 69.

${ }^{23}$ KESSLER, H. Erlösung als Befreiung, S. 54-55.

${ }^{24}$ VÖGTLE, A. Todesankündigungen und Todesverständnis Jesu, S. 69.
} 
Leben schenken kann. Wird Sünde aber innerhalb der Proportionen der menschlichen Existenz aufgefasst, dann können Menschen durchaus dazu beitragen, dass Sünde, nicht nur politisch und gesellschaftsstrukturell, sondern auch existenziell und religiös, überwunden werde.

Nicht von ungefähr stellt deswegen Magnus Striet fest, dass sich bedauerlicherweise die geschichtliche Debatte um die soteriologische Bedeutung des Todes Jesu vorwiegend um die Frage nach der Sündhaftigkeit des Menschen gedreht habe. Er konstatiert weiter: „Bis heute ist die Ansicht in der Theologie weit verbreitet, dass, wenn man nicht von einer Erlösungsbedürftigkeit des Menschen von einer allgemeinen Sünde ausgehe, nicht mehr verstehbar gemacht werden könne, warum Gott Mensch geworden sei." ${ }^{25}$ Die Gefahr, die solche Theologien laufen, ist die Inthronisierung der übermächtigen Kategorie der Sünde auf Kosten der ungeschuldeten, grundlosen, allem zuvorkommenden Gnade Gottes. Sich auf Scotus beziehend weist Karl Rahner eben aus diesem Grund darauf hin, dass die Sünde im Hinblick auf Gottes unbedingte Liebe, die sich in und durch Christus gezeigt hat, schon immer überholt oder nachrangig war. Hätte Gott die Welt nicht auf eine solche radikale Weise geliebt, hätte er die Sünde in der Welt überhaupt nicht zugelassen. ${ }^{26}$

Menke stimmt Striets Thesen in weiten Teilen zu und lehnt ebenso dezidiert jegliche Stellvertretungstheorie ab, welche etwa die anselmsche Satisfaktionslehre wieder beleben möchte, da ein solches Gottesbild antibiblisch sei und Gewalt rechtfertige. ${ }^{27}$ Doch ist für ihn eine Deutung des Golgotha-Ereignisses ausschließlich als Offenbarungsgeschehen der unbedingten Liebe Gottes nicht hinreichend. ${ }^{28}$ Für ihn tut Gott da tatsächlich etwas dem Sünder gegenüber. Am Kreuzesgeschehen gelangt Gott nämlich zu jenem Ort, den er bis zum Tod seines Sohnes und zugleich des Menschen Jesu nicht erreichen konnte, nämlich die Sphäre der Sünde und des Todes, eine Wirklichkeit, welche dem jüdischen Bild der „Scheol“ entsprechen könnte. Diesen Ort konnte Gott zuvor selbst „mit der Kraft seiner barmherzigen Liebe“ deshalb nicht beseitigen,

\footnotetext{
${ }^{25}$ PRÖPPER, Th. Erlösungsglaube und Freiheitsgeschichte, S. 244-245; vgl. VÖGTLE, A. Todesankündigungen und Todesverständnis Jesu, S. 110.

${ }^{26}$ PRÖPPER, Th. Erlösungsglaube und Freiheitsgeschichte, 79.

${ }^{27}$ STRIET, M. Erlösung durch den Opfertod Jesu, S. 13.

${ }^{28}$ Erlösung besagt das Heil des ganzen Menschen; Erlösung liegt der Sündigkeit voraus; Erlösung geschieht in der endgültigen Selbstmitteilung Gottes (vgl. RAHNER, K. Das christliche Verständnis der Erlösung, S. 245).
} 
„[...] weil der Schöpfer mit der Ermöglichung wirklicher Freiheit die Setzung einer Wirklichkeit riskiert hat, über die er im wahrsten Sinne dieses Wortes nicht verfügen kann. “29 Erst durch den Tod des Menschen Jesu kann Gott kraft seiner Beziehung zu seinem Sohn zu diesem Ort gelangen und dem Sünder oder den Toten durch den Sohn eine Hand reichen, damit der Sünder von den Fesseln der Sünde befreit werde.

Genau darin besteht die Sühne der Sünde. Denn Sünde wird von Menke als eine objektive Wirklichkeit aufgefasst, die eine Eigenwirksamkeit besitzt und entfaltet. ${ }^{30}$ Zwar lehnt er mit Striet und Pröpper eine augustinistische Erbsündenlehre, ,die den Zusammenhang zwischen Sünde und Freiheit zerreißt" ${ }^{31}$ ab, doch scheint auch Menke die Sünde als eine schwerwiegendere Kategorie zu bestimmen, vor der der Mensch bloß dastehen und wogegen er vergebens etwas unternehmen kann. Außerdem erscheint das von Menke vertretene Modell der Sühne und Erlösung allzu „dramatisch“ oder von der balthasarschen Theologie geprägt. Denn der Mensch steht vor dieser ihm hingereichten Hand und muss sich entweder dafür oder dagegen entscheiden, er muss entweder die Sünde oder diese Hand zurückweisen. Ob eine solche „einfache Entscheidung“ der Komplexität des Lebens und jeder Beziehung tatsächlich gerecht wird, ist jedoch fraglich.

Selbst wenn man das christliche Heil als Teilnahme an der Beziehung mit dem trinitarischen Gott auffassen möchte, ${ }^{32}$ d.h. als eine „subjektive“ Erlösung, kann man sie nicht als eine EntwederOder-Beziehung denken. Die menschliche Beziehung zu Gott bleibt immer prekär und ambivalent. Doch deswegen hört sie keineswegs auf, Beziehung zu sein. In jeder Liebesbeziehung besteht doch die Kraft der Vergebung und der Liebe gerade in der prozesshaften Wiederherstellung der Beziehung selbst. Will man menschliche Beziehungen ernst nehmen, so gehören die Sünde und das Scheitern unbedingt dazu. Aber dies heißt längst nicht, dass man sich gegen die Beziehung entschieden hat, sondern lediglich, dass man der conditio humana wegen nicht imstande ist, eine Beziehung statisch, unveränderlich und perfekt zu leben. Man lebt eine Beziehung schon immer als Sünder, aber man hofft zugleich, dass man durch die Kraft der Liebe und der Vergebung vom

\footnotetext{
${ }^{29}$ MENKE, K.-H. Gott sühnt in seiner Menschwerdung die Sünde des Menschen, S. 101.

${ }^{30}$ MENKE, K.-H. Gott sühnt in seiner Menschwerdung die Sünde des Menschen, S. 106.

${ }^{31}$ MENKE, K.-H. Gott sühnt in seiner Menschwerdung die Sünde des Menschen, S. 110.

${ }^{32}$ MENKE, K.-H. Gott sühnt in seiner Menschwerdung die Sünde des Menschen, S. 110.
} 
Anderen getragen, bejaht, und angenommen werde, und zwar so wie man ist, unbedingt. Nur eine solche gegenseitige Erfahrung kann eine Beziehung tragen und jedem Partner dieses Bundes Halt und Hoffnung geben. Somit wird Vergebung nicht primär als Tilgung der Schuld, sondern als Ermöglichung eines Neuanfangs verstanden, kraft dessen das Leben seine Wonne zurückgewinnen kann. Erst durch die Kraft solcher Beziehungen kann das Subjekt über den Umweg des Anderen den Weg zu sich selbst finden oder, mit Ricoeur gesprochen, zu einer Hermeneutik des Selbst gelangen. ${ }^{33}$

Damit ist keineswegs eine Verharmlosung oder Leugnung der Sünde in der menschlicher Existenz gemeint, sondern es wird vielmehr ihre Rolle innerhalb von Beziehungen hervorgehoben. Wenn jedoch sie menschliche Dimensionen übersteigt oder sich vom Menschen verselbständigt, dann muss sie tatsächlich zum Grund göttlicher Offenbarung und Inkarnation werden und gerade deswegen zur Bedingung Gottes absoluter Freiheit. Zur Folge hat eine solche Bedingung natürlich eine Verdunklung und warum auch nicht eben eine Infragestellung der allem zuvorkommenden Gnade Gottes. Die Frage wird hierzu von Bedeutung sein, wo man Parameter findet, um mit der Kategorie der Sünde angemessen umzugehen.

Letztendlich bringen die Art und Weise, wie Jesus vergeben oder geheilt hat, der in den Evangelien ein hohes Maß an soteriologischer Bedeutung beigemessen wird, oder seine Botschaft über Heil und Vergebung eines zum Ausdruck: wieder integriert sein in das Reich des Seins, in das Reich Gottes, in die Fülle des Lebens. Jede Heilung oder Vergebung der Sünde enthält die verheißungsvolle, Leben ermöglichende Aussage: „Du bist und wirst nicht mehr ausgeschlossen von der Gemeinschaft, vom Seindürfen. Dein Lebensentwurf erscheint, warum auch immer, gescheitert. Doch darfst du weiter leben." Wenn die theologische Auslegung des Todes Jesu nicht in Übereinstimmung mit dieser frohen Botschaft geschieht oder wenn sie sogar das Gegenteil bewirkt etwa durch Exklusion oder die Lehre einer prädestinierten Auserwählung einiger Weniger, muss sie sicher einer Revision unterzogen werden.

Wenn der sühnende Tod Jesu als der alleinige Maßstab der Versöhnung Gottes mit dem Menschen zu verstehen wäre, welche Kriterien könnten dann noch herangezogen werden, fragt Striet

${ }^{33}$ MENKE, K.-H. Gott sühnt in seiner Menschwerdung die Sünde des Menschen, S. 108. 
zurecht, um eine „Hypostasierung des Schmerzens“, eine „Verklärung des Leids bzw. des Martyriums" zu unterbinden, welche er in Anlehnung an Kermanis Überlegungen über das Recht und die Verantwortlichkeit der Rede über Gott als barbarisch, körperfeindlich und ungerecht oder undankbar dem Schöpfer und der Schöpfung gegenüber empfindet. ${ }^{34}$

Wenn allein die kerygmatische Verkündigung des Todes Jesu bleiben soll, was könnte einem paulinischen, patrozentrischen, ja monotheistischen Gottesbild im Wege stehen, dem zufolge allein der Vater Subjekt und Ökonom des Heilsplans für die Menschheit wäre und Jesus bloß ein treuer, gehorsamer Vollzieher dessen? Wie kann man da die unbedingte Freiheit des Menschen Jesu noch retten? ${ }^{35}$

Der Allmächtige bestimmt sich dazu, sich von der durch ihn selbst geschenkten Freiheit des Menschen nicht nur scheinbar, sondern mit allen Konsequenzen bestimmen zu lassen; das heißt, Gott verpflichtet sich in souveräner Freiheit dazu, sich mit keinen anderen Mitteln gegen den einzelnen Menschen oder die Geschichte insgesamt durchzusetzen als mit denen der unbedingen Anerkennung der einmal geschenkten Andersheit der geschöpflichen Freiheit. Deshalb ist das Kreuz Jesu Christi die Konsequenz einer Bejahung der Andersheit des Sünders, die sich lieber von diesem foltern läßt als ihn zu der von ihr erhofften Gegenliebe zu zwingen. Der Inhalt des erlösten Menschseins, nämlich die Liebe, läßt sich nicht erzwingen, auch von Gott nicht. Denn Liebe ist der Inhalt selbstursprünglicher Freiheit, oder sie ist gar keine Liebe. ${ }^{36}$

\subsection{Der umstrittene Stellvertretungsgedanke}

Demgegenüber versuchen andere Theologien diese selbe Liebe Gottes mithilfe des Stellvertretungsmodell zu verdeutlichen. So entfalten neuere Ansätze in dieser Richtung wie beispielsweise Karl-

${ }^{34}$ KASPER, W. Jesus der Christus, S. 376; SATTLER, D. Beziehungsdenken in der Erlösungslehre; GRESHAKE, G. Gottes Heil, Glück des Menschen; MENKE, K.-H. Gott sühnt in seiner Menschwerdung die Sünde des Menschen, S. 111-113.

${ }^{35}$ Hierzu vgl. die theologische Studie von Knut Wenzel zu Ricoeurs hermeneutischem Ansatz über die christliche Kategorie der Vergebung: WENZEL, K. Glaube in Vermittlung, bes. Kap. X (Anerkennung des Anderen angesichts der Schuld?); s. auch RICOEUR, P. Wege der Anerkennung, bes. Kap. V (Der Kampf um Anerkennung und die Friedenszustände).

${ }^{36}$ STRIET, M. Erlösung durch den Opfertod Jesu?, S. 11; vgl. auch KERMANI, N. Bildansichten. 
Heinz Menkes ihre Theorien im Rahmen einer Bundestheologie. ${ }^{37}$ Für Menke betrifft ein solches bundesgemäßes Handeln besonders Gottes Handeln gegenüber dem Sünder. „Jahwes Verzeihen besteht nicht in einer Aufhebung der Vergangenheit des Sünders, sondern im Gewähren einer Zukunft, die der reuige Sünder selbst realisieren muss, indem er an die ihm von Gott gewährte Stelle im Bundesverhältnis zurückkehrt.“"38 Die Initiative erfolgt demnach allein vonseiten Gottes. Denn, wenn die Geschichte nicht das Weltgericht sein soll, wie Schiller gesagt hat, wenn die Sünde in der Welt überwunden werden soll, dann kann Gottes Bundeshandeln kein bedingtes, sondern es muss ein unbedingtes Handeln sein. Er muss an Stelle des Sünders getreten sein, damit allen Sündern dadurch vergeben werden kann. „Nur wenn der Gekreuzigte nicht allein Mensch, sondern zugleich der mit dem ewigen Wort des Vaters geeinte Sohn gewesen ist, kann sein Sterben die rettende und versöhnende Kraft gehabt haben die ihm die Kirche von Anfang an zuerkannt hat.“39

Damit soll nach Jan-Heiner Tück Gottes unbedingte Solidarität sowohl mit den Opfern als auch mit den Tätern der Geschichte zum Ausdruck gebracht werden, in der Hoffnung, dass die Täter ihre Taten bereuen und die Opfer ,[...] vergeben, was ihnen nicht zurückgegeben werden $\operatorname{kann}[\ldots]^{640}$ und dadurch die universale Versöhnung geschehen mag. Auch Vechtel greift am Ende seines Aufsatzes auf Rahners „Übersetzung“ des ,problematischen Begriff[s] der Stellvertretung“ als „unbedingter Solidarität"“41 für die Menschheit zurück und setzt den einen mit dem anderen gleich, um womöglich den Stellvertretungsgedanken

${ }^{37}$ Dabei soll daran erinnert werden, das dies nicht ausschließlich ein modernes Anliegen im theologischen Diskurs ist. Der Streit gegen den Monotheletismus im 7. Jahrhundert, der 681 mit dem 3. Konzil von Konstantinopel beendet wurde, zeigt in aller Deutlichkeit, wie sehr die Freiheit des Menschen Jesu sicherzustellen ist, selbst bei seinem grausamen Tod. Die Lehre eines der Hauptstreiter gegen den Monotheletismus, des Maximus Confessor, zeigt, dass die Freiheit Jesu selbst in diesem verhängnisvollen Geschehen keineswegs zweitrangig sei oder im Willen des Vaters untergehe. Außerdem lässt sich der Menschensohn in souveräner Freiheit nicht nur von Gott bestimmen, sondern auch von seinen geschichtlichen und partikularen Interaktionen. Vgl. hierzu McFARLAND, I. Willing Is Not Choosing.

${ }^{38}$ So fasst Menke Pröppers soteriologisches Anliegen zusammen (MENKE, K.-H. Stellvertretung - Befreiung - Communio, S. 56-57).

${ }^{39}$ VECHTEL, K. Musste Jesus für uns leiden?, S. 351.

${ }^{40}$ So fasst Vechtel Menkes Gedanken zusammen (VECHTEL, K. Musste Jesus für uns leiden?, S. 351).

${ }^{41}$ TÜCK, J.-H. Am Ort der Verlorenheit, S. 49. 
doch noch retten zu können. Auch wenn Vechtel auf subjekttheoretische Fragen eingeht, reicht seine Begründung nicht aus, um deutlich zu machen, warum die menschliche ,Situation von Unheil, von Schuld und Rivalität" durch eine unbedingte Solidarität zunichte gemacht werden solle. ${ }^{42}$ Letztendlich besteht Jesu Solidarität in der Annihilierung der Sünde oder darin, „[...] seine ihm vorgegebene Solidarität mit den Menschen überhaupt auch dort noch in freier Liebe durch[zu]halten und nicht auf[zu]kündigen, wo sie für ihn das Kreuz und den Tod in Gottverlassenheit bedeutete[?]“43

Die an dieser Stelle auftretende Rückfrage an Stellvertretungstheorien wäre dann, ob dieses unbedingte Handeln Gottes in der Geschichte tatsächlich den intendierten Effekt auf die Sünder und Täter hat. Es geht also um die Frage nach der Wirkung des Todes Jesu oder danach, inwiefern der Tod Jesu den heutigen Menschen noch betreffen oder erreichen kann. Rahner begegnet solchen Ansätzen mit folgender Einstellung:

Eine Konzeption der stellvertretenden Erlösung, in der Jesus an meiner Stelle etwas tut, was ich eigentlich tun sollte, aber nicht kann und was dann mir ,angerechnet“ wird, halte ich für falsch oder mindestens für eine mißverständliche Formulierung der dogmatischen Wahrheit, daß meine Erlösung von Jesus und seinem Kreuz abhängig ist. ${ }^{44}$

Kein Zweifel kann aufgeworfen werden, dass Gott und Jesu Leben, Botschaft und Geschick den Prozess der Erlösung erst konstituieren. Doch kann er nicht Gott allein überlassen werden, wenn der Mensch und die gesamte Schöpfung, welche Gott so bedingungslos liebt, ernst zu nehmen sind. Selbst wenn von Balthasar, Tück und andere zeitgenössische Vertreter der Stellvertretungstheorie argumentieren mögen, dass Jesus den Sünder nicht „beiseite schieben“ kann, dass er den Sünder nicht aus seiner Gefangenschaft entfesseln kann, ohne dass er diese Erlösungstat aus tiefer Dankbarkeit annimmt, ohne dass er sie für sich selbst „,wahr sein lässt"“, ${ }^{45}$ bleibt die Frage offen, ob der Mensch noch Mensch bliebe, wenn er von der Macht der Sünde tatsächlich befreit würde bzw. was dieses magische Geschehen im Leben des Gläubigen de

\footnotetext{
${ }^{42}$ WERBICK, J. Gott verbindlich: eine theologische Gotteslehre, S. 511.

${ }^{43}$ RAHNER, K. Versöhnung und Stellvertretung, S. 262.

${ }^{44}$ Vgl. VECHTEL, K. Musste Jesus für uns leiden?, S. 357.

${ }^{45}$ RAHNER, K. Versöhnung und Stellvertretung, S. 263.
} 
facto bewirkt. Mit anderen Worten muss offen bleiben, ob die Tatsache, dass jemand für mich etwas tut, was ich nicht tun kann, tatsächlich so heilbringend sein kann, wie man es verkündet. Es stellt sich die Frage, ob es nicht mit dem von Jesus hinterlassenen Zeugnis konformer ist zu glauben, dass man von Gott dem Vater unbedingt geliebt ist trotz der Sünde? Letztendlich muss man Rahner zustimmen, ,daß das Erbarmen Gottes, wenn es will, die Schuld der menschlichen Freiheit nicht beseitigen kann, ohne sie aufzuheben, so daß also die letzte Frage über eine mögliche Verdammnis und endgültige Unerlöstheit doch wieder an die souveräne Verfügung Gottes gerichtet werden muß.““46

\subsection{Das Kreuzesgeschehen als signum efficax}

Das problematische, von Rahner kritisierte Verständnis des erlösenden Werks Jesu verdankt sich nach Greshake der Tatsache, dass Gnade seit Augustinus und seinem Sieg über den Pelagianismus ausschließlich als ,innere Gnade“ aufgefasst wird. ${ }^{47}$ Damit ist eine unmittelbare Beziehung zwischen Gott selbst und dem Menschen und der damit einhergehende Verzicht jeglicher Vermittlung gegeben. Geschichtlich führte dies zu einer Unterscheidung zwischen objektiver und subjektiver Erlösung, deren Folge eine Entzweiung von Christologie und Soteriologie war. Die Beziehungslosigkeit der Gnade und der Erlösung zur geschichtlichen Realität, zur civitas terrena, und der Relevanzverlust der Christologie veranlassten eine progressive Entfremdung des Glaubens dem Menschen gegenüber sowie eine Art Entzug hinsichtlich der eigenen Verantwortung für das eigene Heil und für das der Welt. Vor diesem Hintergrund ist die „Säkularisierung der Soteriologie" der Neuzeit ${ }^{48}$ sowie die besondere Gewichtung der menschlicher Praxis bei den soteriologischen Entwürfen der politischen oder der Befreiungstheologie zu verstehen.

Will man dennoch den soteriologischen Sinn des Todes Jesu als erlösendes Geschehen begreifen, das viel mehr als bloß eine „moralisch effiziente Ursache“ $\mathrm{zu}$ meinen scheint, vor allem in Hinblick auf seine Bedeutung für den Gläubigen, so müsste man, so Rahners Vorschlag, das Kreuz als signum efficax, also als Realsymbol oder wirksames Zeichen der erlösenden Liebe Gottes in der Welt

\footnotetext{
${ }^{46}$ RAHNER, K. Das christliche Verständnis der Erlösung, S. 244-45.

47 BALTHASAR, H. U. Epilog, S. 95; TÜCK, J.-H. Am Ort der Verlorenheit, S. 49.

${ }^{48}$ RAHNER, K. Das christliche Verständnis der Erlösung, S. 246.
} 
auffassen. ${ }^{49}$ Das Kreuz als Zeichen der grundlosen Liebe und der unbedingten Freiheit Gottes zu deuten, leuchtet einem ein. Wie kann man aber zugleich die Glaubensaussage des Todes Jesu als Ursache für das Heil aller denken, fragt sich Rahner? Zur Beantwortung dieser Frage fügt er Kreuz und Auferstehung zusammen und präzisiert seine Begrifflichkeit. Er spricht von einer ,sakramentale[n] Zeichenursächlichkeit“, ,[...] die vom ihr vorausgehenden Heilswillen Gottes selber gesetzt, nicht [aber] die Ursache dieses Heilswillens ist. " ${ }^{50}$ Der Tod Jesu wird als Offenbarung Gottes gedacht und betrifft tatsächlich alle Menschen, sofern das Heil ohne Vorbedingungen allen zugänglich wird. Auf Golgotha manifestiert sich deswegen Gottes unbedingte Liebe als zeichenhaftes, sakramentales, wirksames Geschehen. Gerade kraft seiner konkreten Partikularität kann die Wirkung dieses Zeichens als universal bezeichnet werden, wenn dies mehr als bloße Behauptung sein will. Denn der Inhalt der Offenbarung, d.i. Gottes entschiedene Liebe zum Menschen, und ihre Gestalt, d.i. die geschichtlich symbolische Vermittlung, gehören dermaßen untrennbar zusammen, dass der Inhalt der Vermittlung bedarf, ,[...] um überhaupt Wahrheit und Wirklichkeit für uns Menschen zu werden." Somit folgert Pröpper, dass, ,[...] wenn die symbolische Handlung ernsthaft als Ereignis ihrer bedeuteten Wahrheit gedacht [...] und der Anteil der Freiheit Jesu an ihm nicht übersehen wird, der Tod Jesu [...] der Vollzug der Erlösung [ist]. " ${ }^{61}$ Nur auf diese Weise kann die behauptete Liebe nicht als bloßes Ideal verstanden, sondern als reales Geschehen wahrgenommen und sogar erfahren werden.

\section{$5 \quad$ Ergebnis}

Zusammenfassend lässt sich als Ergebnis der vorherigen Überlegungen festhalten, dass der Tod Jesu, begriffen als Heilsgeschehen, so bedeutsam er für den christlichen Glauben sein mag, nie getrennt oder unabhängig vom Leben, von der Botschaft, der Praxis und der Auferstehung Jesu gedacht werden darf. Seine soteriologische Bedeutung lässt sich vielmehr von Gottes ,gesamter Offenbarung“ her erschließen, welche als grundlose Mitteilung der Liebe aufzufassen

\footnotetext{
${ }^{49}$ Vgl. GRESHAKE, G. Gnade als konkrete Freiheit; PRÖPPER, Th. Erlösungsglaube und Freiheitsgeschichte, S. 101-102.

${ }_{50}$ PRÖPPER, Th. Erlösungsglaube und Freiheitsgeschichte, 102.

51 „Ursache, weil wirksames Zeichen, weil exhibitives Realsymbol; Zeichen, weil durch das Bezeichnete verursacht (RAHNER, K. Das christliche Verständnis der Erlösung, S. 246).“
} 
ist. Die unterschiedlichen Metaphern des Neuen Testaments für den heilsamen Tod Jesu sind zweifelsohne bedeutungsvolle verbindliche Interpretationsversuche eines grausamen Geschehens, das für den Glauben konstitutiv ist. Doch erschöpfen sie in keiner Weise das Bedeutungspotential dieses Geschehens und dürfen mit Berechtigung insofern verwendet werden, als sie in Vereinbarkeit mit dem gesamten Leben und der gesamten Botschaft Jesu stehen und zugleich mit den Bedingungen und Möglichkeiten der geschichtlichen, menschlichen Vernunft, die es interpretiert. Die ,ausführliche Einleitung“ der Evangelien auf Jesu Passionsgeschichte hin (Martin Kähler) darf in keiner Weise lediglich als „dekoratives Beiwerk“ angesehen werden. Vielmehr bestimmt die Erzählung des Lebens des irdischen Jesu die Interpretation seines Todes mit.

Dabei ist die theologische sowie anthropologische Erschließung der Kategorie der Sünde von ganz entscheidender Bedeutung, da sie den soteriologischen Diskurs übermäßig bestimmen kann. Der sühnende Tod Jesu am Kreuz stellt keine neue oder andere Art der Vergebung der Sünde dar als jene des irdischen Jesu. Er, der Tod, kann die jesuanische Art der Vergebung bestenfalls radikalisieren, nicht aber überbieten oder gar ersetzen. Anders als ein magisches Geschehen ist die geglaubte, durch den Opfertod geleistete Tilgung der Sünde eine Ermöglichung zu verwandeltem, ja neuem Leben. Im Vordergrund stehen deshalb weder die Tilgung selbst noch die Tat, sondern die Begegnung Gottes mit seiner Schöpfung bzw. mit dem Sünder. So gesehen muss angezweifelt werden, ob der Sinngehalt jeglicher Hypostasierung des Leids, des Schmerzes und des Opfers tatsächlich christlich und ursprünglich ist. Das rechte Verständnis des Todes Jesu kann in der Tat zur vera icona dei führen, zur vera fides. Verkürzte oder verengte Deutungen dieses Geschehens vermitteln hingegen problematische Gottesbilder, die zu einer dezidierten Ablehnung Gottes führen können, anstatt Heil, neues, ewiges Leben zu schenken.

\section{Bibliographie}

BALTHASAR, Hans Urs von. Epilog. Einsiedeln; Zürich; Köln: Johannes, 1987.

BARTH, Gerhard. Der Tod Jesu Christi im Verständnis des Neuen Testaments. 2. Aufl. Neukirchen-Vluyn: Neukirchener, 2003.

EBELING, Gerhard. Der Sühnetod Christi als Glaubensaussage: Eine hermeneutische Rechenschaft. Zeitschrift für Theologie und Kirche, Tübingen, Beiheft 8, 1990, S. 3-28. 
GRESHAKE, Gisbert. Gnade als konkrete Freiheit: eine Untersuchung zur Gnadenlehre des Pelagius. Mainz: Matthias Grünewald, 1972.

. Gottes Heil, Glück des Menschen: theologische Perspektiven. Freiburg im Breisgau: Herder, 1983.

KASPER, Walter. Jesus der Christus. Gesammelte Schriften 3. Freiburg im Breisgau; Basel; Wien: Herder, 2007.

KERMANI, Navid. Bildansichten: Warum hast du uns verlassen? Neue Zürcher Zeitung. Zugegriffen 13. Februar 2016. <http://www.nzz.ch/warum-hast-du-unsverlassen--guido-renis-kreuzigung-1.2195409>.

KESSLER, Hans. Erlösung als Befreiung. Düsseldorf: Patmos, 1972.

McFARLAND, Ian A. 'Willing Is Not Choosing': Some Anthropological Implications of Dyothelite Christology. International Journal of Systematic Theology, Bd. 9, Nr. 1, S. 3-23, Januar 2007. doi:10.1111/j.1468-2400.2006.00227.x.

MENKE, Karl-Heinz. Gott sühnt in seiner Menschwerdung die Sünde des Menschen. In: STRIET, Magnus; TÜCK, Jan-Heiner (Hg.) Erlösung auf Golgota? Der Opfertod Jesu im Streit der Interpretationen. Theologie kontrovers. Freiburg im Breisgau; Basel; Wien: Herder, 2012, S. 101-125.

. Stellvertretung - Befreiung - Communio: Die zentralen Denkformen der Soteriologie in der deutschsprachigen Theologie des 20. Jahrhunderts. Theologie und Philosophie, Frankfurt am Main, Bd. 81, Nr. 1, S. 21-59, 2006.

PRÖPPER, Thomas. Erlösungsglaube und Freiheitsgeschichte: Eine Skizze zur Soteriologie. 3. Aufl. München: Kösel, 1991.

RAHNER, Karl. Das christliche Verständnis der Erlösung. In: Ders. Schriften zur Theologie, 15. Zürich; Einsiedeln; Köln: Benziger, 1983, S. 236-250.

. Der eine Jesus Christus und die Universalität des Heils. In: Ders. Schriften zur Theologie, 12. Zürich; Einsiedeln; Köln: Benziger, 1975. S. 251-282.

. Versöhnung und Stellvertretung. In: Ders. Schriften zur Theologie, 15. Zürich; Einsiedeln; Köln: Benziger, 1983. S. 251-264.

RICOEUR, Paul. Wege der Anerkennung. Erkennen, Wiedererkennen, Anerkanntsein. Frankfurt am Main: Suhrkamp, 2006.

SATTLER, Dorothea. Beziehungsdenken in der Erlösungslehre: Bedeutung und Grenzen. Freiburg: Herder, 1997.

STEYN, Gert J. Soteriological Perspectives in Luke's Gospel. In: WATT, Jan Gabriel van der (Hg.) Salvation in the New Testament: Perspectives on Soteriology. Supplements to Novum Testamentum 121. Leiden: Brill, 2005. S. 67-99.

STRIET, Magnus. Erlösung durch den Opfertod Jesu? In: STRIET, Magnus; TÜCK, JanHeiner (Hg.) Erlösung auf Golgota? Der Opfertod Jesu im Streit der Interpretationen. Theologie kontrovers. Freiburg im Breisgau; Basel; Wien: Herder, 2012, S. 11-31. 
STUDER, Basil. Gott und unsere Erlösung im Glauben der Alten Kirche. Düsseldorf: Patmos, 1985.

TÜCK, Jan-Heiner. „Am Ort der Verlorenheit: Ein Zugang zur rettenden und erlösenden Kraft des Kreuzes“. In: STRIET, Magnus; TÜCK, Jan-Heiner (Hg.) Erlösung auf Golgota? Der Opfertod Jesu im Streit der Interpretationen. Theologie kontrovers. Freiburg im Breisgau; Basel; Wien: Herder, 2012. S. 33-58.

VECHTEL, Klaus. Musste Jesus für uns leiden? Soteriologie und Gottesbild. Theologie und Philosophie, Frankfurt am Main, Bd. 89, Nr. 3, 2014, S. 341-59.

Vögtle, Anton. Todesankündigungen und Todesverständnis Jesu. In: BEUTLER, Johannes; KERTELGE, Karl (Hg.) Der Tod Jesu: Deutungen im Neuen Testament. Quaestiones disputatae 74. Freiburg im Breisgau; Basel; Wien: Herder, 1976. S. 51-113

WENZEL, Knut. Glaube in Vermittlung: theologische Hermeneutik nach Paul Ricoeur. Freiburg im Breisgau; Basel; Wien: Herder, 2008.

WERBICK, Jürgen. Gott verbindlich: eine theologische Gotteslehre. Freiburg im Breisgau: Herder, 2007. . Soteriologie. Leitfaden Theologie 16. Düsseldorf: Patmos, 1990.

Recebido em: 29/05/2016

Aprovado em: 16/07/2016 